\title{
A Review of some Pure-Pursuit based Path Tracking Techniques for Control of Autonomous Vehicle
}

\author{
Moveh Samuel \\ Department of Mechanical \\ Engineering, Faculty of \\ Mechanical Engineering \\ Universiti Tecknologi Malaysia \\ Johor Bahru, Johor Malaysia.
}

\author{
Mohamed Hussein \\ Intelligent control and \\ automation (ICA) Research \\ group department of \\ Mechanical Engineering, \\ Faculty of Mechanical \\ Engineering Universiti \\ Tecknologi Malaysia Johor \\ Bahru, Johor Malaysia.
}

\author{
Maziah Binti Mohamad \\ Department System Dynamic, \\ Control and Design, Faculty of \\ Mechanical Engineering \\ Universiti Teknologi Malaysia \\ 81310, UTM Johor Bahru, \\ Johor, Malaysia
}

\begin{abstract}
This paper gives a brief review of few common path tracking techniques used in the design of autonomous vehicles. Technique such as pure-pursuit, vector pursuit as well as CFpursuit which are all based on the pure-pursuit techniques were discussed and a detailed comparism was made between these geometric techniques. Also this review work discusses areas were little research has been done. Areas such as tracking of an implicit part of a mobile robot and proposes an area where feature research can be done such as tracking of both implicit and explicit path for a non-holonomic mobile robot.
\end{abstract}

\section{Keywords}

Autonomous vehicle, path-tracking, pure-pursuit, sensors, controller, implicit, explicit.

\section{INTRODUCTION}

An autonomous vehicle is a self-driven vehicle that drive itself with necessary sensors, such as GPS, IMU, cameras, sensors etc. The basic operational process is such that the vehicle first detects the environment and positions itself according to these sensors, and then navigates itself with global and local planner; finally, the vehicle drives its self autonomously by executing the necessary control command along the given path. The path of a mobile robot is described as the route that the vehicle would follow in an environment. It is very important in carrying out mobile robot mission. Path tracking controllers are used to carry out the path following operations and minimal lateral distance as well as the heading between the vehicle and defined path is achieved by a good path-tracking controller [1]. Their goal basically is to autonomously navigate and drive the robot along the path by continually generating speed and steering commands which compensate for the tracking errors, which mainly consist of vehicle's deviations in distance and heading from the path. Feedback and feedforward control mechanisms are used for this purpose, with a tradeoff between control effort and control error. Some examples of path tracking techniques for autonomous ground vehicles are based on nonlinear control theory, such as Predictive-Control [2] or Fuzzy-Control [3]. Alternatively, simpler tracking strategies are achieved by geometric considerations between a current vehicle position and the path to follow [4]. Pure-pursuit algorithm is the most common and effective geometric method, which is used to calculates the current position of the vehicle and a set point in the path. This point is chosen at a specified look-ahead distance, which is the chord length of this arc. Some of the benefits of this method include tuning ease of the look-ahead distance, computational simplicity, and the absence of derivative terms.

\section{CLASSIFICATION OF PATH OF MOBILE ROBOT}

There are two main broad classification of paths of a mobile robot: explicit or implicit. An explicit path is described by either of the following: as a sequel of way-point coordinates that are joined by straight line segments or by controlling a parametric curve [5]. Here computation of tracking error involves real-time calculation of the position of the vehicle with respect to the path. However, this basically implies that processing signals from various sensors and relating them with a geometric model of the environment [6]. While implicit path is defined by perceivable features in the environment with an appropriate set of sensor, basically a camera. Examples given by other researchers includes: A route determines a path that is recognized as an image by its left and right edges [7], an object course can be detected as point clusters in consecutive range scans [8]. Therefore, computational tracking error with respect to an implicit path does not require global position calculations, but rather the path is determined by the processing of the images taken by the camera.

The basic idea of reactive navigation is that the only essential data for a particular path needs to be read from sensor data [9], this way, it is possible to simplify processing complexity to a great extent. Not neglecting the fact that some problems have to be coped with to implement reactivity. Initially, dependence on sensor data can lead to a shaky response. Secondly, non-holonomic constraints limit the possible movements from a given position. Thirdly, current mobile robot sensors can provide a large amount of information that needs to be timely processed.

\section{LITERATURE REVIEW GEOMETRIC PATH TRACKING TECHNIQUES}

\subsection{Pure-pursuit}

Pure-pursuit can be dated back in history to the pursuit of missile to a target [10]. In this process, the missile velocity vector is always directed toward the instantaneous target position. Wallace et al in 1985 were the first to develop purepursuit strategy in the field of robotics, were they developed a method for estimating the steering necessary to maintain the vehicle on the road [7]. They achieved this by keeping the 
road centered in the image obtained from an onboard camera mounted on the vehicle. It was based on this concept that Amidi proposed a pure-pursuit method that follows explicit path [4].

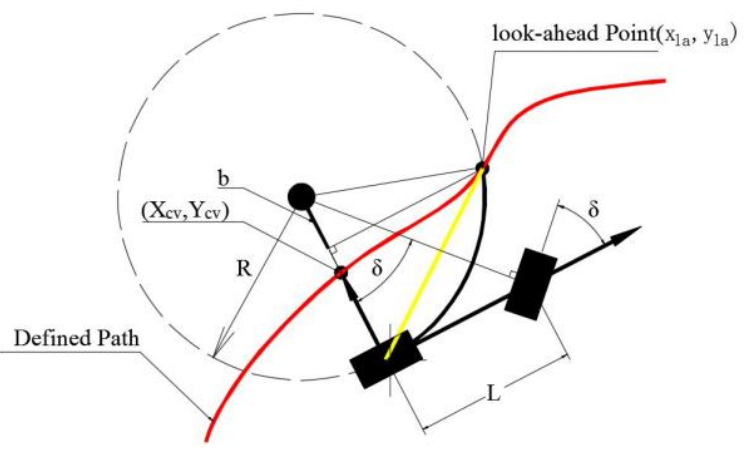

Figure 1: Geometric explanation of pure-pursuit

From figure one above the pure-pursuit process can be implemented as detailed below

1. Find the current location of the vehicle in the global coordinate system ( $\left.x_{\text {vehicle }}, y_{\text {vehicle }}\right)$;

2. 2. Find the closest point on the path to the vehicle, $(X c v, Y c v)$ which is used to locate the vehicle on the path, at which point we can search from it;

3. Choosing a constant look-ahead distance and thensearch the goal point $\left(X_{l a}, Y_{l a}\right)$;

4. Transform the goal point to vehicle coordinates $\left(x_{l a}, y_{l a}\right)$;

5. Calculate the curvature and then acquire the steering angle from Equation (1);

6. Update the vehicle's position and recycle.

Error $_{\text {cte }}=$ Error $_{\text {calculate }}+$ Error $_{\text {tracking }}$

The word pure-pursuit implies imagining a vehicle following or chasing a point on given path some distance ahead of it. Seeing the success of the pure-pursuit path tracking algorithm method Coulter [11] in 1992 discussed the implementation issues of pure-pursuit algorithm and since then the purepursuit strategy has been used in many applications for explicit path tracking, for both indoor and outdoor navigation [12], . Murphy [13] handled the stability condition of purepursuit algorithm, by studying the effect of time delays associated with the visual processing for following straightline roads Besides a detailed research of the stability for tracking explicit paths at constant curvature was done taking into account computing, communication and actuators delays in the control loop [14]. Ollero et al. [12] introduced the supervision of pure-pursuit parameters as a real-time fuzzy controller that automatically tunes the look-ahead distance based on path characteristics, velocity, and tracking errors. Rodriguez-Castano et al. [15] presented a fuzzy-supervised pure-pursuit controller for driving big autonomous vehicles at high speed above $80 \mathrm{Km} / \mathrm{h}$ along explicit paths using differential GPS data. While Martınez et al. [16] in the quest to avoid interunit collisions in a vehicle that pulls multiple passive trailers he proposed the application of curvature limitations to the pure-pursuit path tracker. Since pure-pursuit has been the most common method used it is considered as a reference for path tracking strategies. Researchers like Hellstron et al in 2006 [17] did a comparison of both pure- pursuit with the follow-the-past algorithm which uses steering angle as well as curvature of the recorded explicit path. Also Gockley et al. [18] presented a comparison of both the reactive potential-field method and pure-pursuit taking into account a recorded person positions as regarding personfollowing with a 2D laser scanner. Some further improvement on pure-pursuit tracking were proposed in tackling some problems Petrinec et al [19] in 2003 solve the problem of vehicle being far away from the path by creating a virtual goal point at the look-ahead distance. Urmson et al in 2006 [20] used an integral correction reducing systematic tracking errors due to variations between desired and actual steering angles to augment the basic pure-pursuit tracker. Proportional term to the heading error between the vehicle and the path was added by Stentz et al. [21]. In addition to the pure-pursuit geometric tracking methods, several other researchers proposed various forms of tracking, some of which were briefly discussed in this paper. Using adaptive PID controller to track predefined paths was proposed by Pan Zhao [22]. Also S-J Huang and G$\mathrm{Y}$ Lin [23] proposed a fuzzy controller used for tracking the path used to finish reverse direction auto-parking maneuvers. The MPC controller which runs online to track a planned path was made by Awais [24], which had the capability of avoiding obstacles. Despite the accuracy these controller possess, certain anomalies are inherent, PID controllers always suffer from the optimization of parameters and overshot in tracking; the fuzzy controllers need more information, and MPC will have high demand in computational resources to get a better result.

\subsection{Vector pursuit}

Another geometric path tracking technique used in tracking the path of a non-holonomic autonomous ground vehicle is vector pursuit. Wit et al [25] presented a work on the control of non-holonomic autonomous ground vehicle as it tracks a given path. They introduced a path-tracking technique known as vector pursuit, which is based on the theory of screws, by Sir Robert Ball in 1900. It generates a desired vehicle turning radius based on the vehicle's immediate location relative to the position of a point ahead on the planned path and the desired orientation along the path at that point. They were of the opinion that vector pursuit being a new geometric path tracking method which uses the theory of screws is similar to other geometric methods in that a look-ahead distance is used to define a current goal point, after which geometry is used to determine the desired motion of the vehicle. However, they noted that vector pursuit is different from other geometric path tracking methods, such as follow-the-carrot or pure pursuit, which do not necessarily depend on the desired orientation of the vehicle at the look-ahead point Also their work indicated that proportional path tracking is a geometric method that does use the desired orientation at the look-ahead point, which adds the current position error multiplied by some gain to the current orientation error multiplied by some gain, and therefore becomes geometrically irrelevant since terms with different units are added. Finally, they concluded that vector pursuit uses both the location and orientation of the look-ahead point while remaining geometrically meaningful.

Sir Robert Stawell Ball Screw theory involves using a screw to explain the instantaneous movement of a body relative to a given coordinate system. This screw used to explain the instantaneous motion of body is known as instantaneous screw. Therefore, it is possible to use screw theory to represent the motion of an autonomous ground vehicle (AGV), i.e., assuming the AVG as the rigid body, from its immediate location and orientation to a desired location and 
orientation that is on a given path. Since screws have centerline that are defined in a given coordinate system and a pitch. Therefore, the instantaneous motion of a rigid body can be illustrated as the body attached to a screw and rotating about that screw at a particular angular velocity at any given instant. Plucker line co-ordinates are used can be used to defined centerline of a screw.

\subsection{CF-pursuit}

Shan et al [14] introduced a new path tracking pursuit technique called CF-pursuit which was based on pure-pursuit with certain differences. In their method they replaced the circles employed in pure-pursuit with clothiod $\mathrm{C}$ curve so as to reduce fitting errors. They used a fuzzy system to consider the path's curvature directly as regarding the choice of lookahead distance. They used 3 variable input in the fuzzy system ( $6 \mathrm{~m}, 9 \mathrm{~m}$ and $12 \mathrm{~m}$ curvatures respectively), estimated from the clothiod fit with the vehicles present position and the goal position on the given path. They choose a sugeno fuzzy model to output a reasonable look-ahead distance using the experiences of real human drivers as well as their tests and compared with some geometric controllers, they concluded that the method of using CF-pursuit performed better in cross track error, stability and robustness and finally based on the result of their field tests carried out, it showed that CF-pursuit is an efficient geometric path tracking techniques for selfdriving cars.

\section{DISCUSSION}

In summary, which so much attention that has been given to pure-pursuit tracking of explicit path by so many researchers, very little interest has been given to tracking of implicit path since the method was introduced into mobile robots [26], except for the work done on a person following with a rotary sonar [27] and a 2D laser scanner where Morales et al [1] investigated pure-pursuit path tracking for reactive tracking of an implicit path with a non-holonomic vehicle, there method was developed to follow obstacles like; walls, persons and corridors based on onboard 2D laser scanner for each. However, since their basic idea of reactive tracking was essentially that, the path to be followed would be read from the camera, which reduces complexity of data processing greatly. Although certain issues have to be dealt with at the initial stage to implement reactivity: firstly, poor response can result from over-dependence on sensor data, non-holonomic hindrances can limit movement from a given position and finally, since mobile robot sensors have abilities to provide huge amount of information, this information have to be timely processed. But by combining both tracking techniques of the implicit and explicit path, the over-dependence of data from the sensors will be reduced by the introduction of a camera which would server as the main eye of the vehicle, the non-holonomic hindrance will be taken care of by computing the tracking error, which involves real-time calculation of vehicles position with respect to the path, taking into account obstacle avoidance such as persons, wall and corridors, Given the other sensors adequate time to process the data read timely.

\section{CONCLUSION}

This work reviews literature and identifies important path tracking models from the vast background and resources. The paper augments the literature with a comprehensive collection of important path tracking ideas, a guide to their implementations and, most importantly, an independent and realistic comparison of the performance of these various approaches.
Therefore, from the above discussion it can be concluded that the combination of both tracking techniques, that is implicit and explicit will path help in reducing the over-dependence of data from the sensors, as well take care of the nonholonomic hindrance by computing the tracking error, which involves real-time calculation of vehicles position with respect to the path, taking into account obstacle avoidance such as persons, wall and corridors, Given the sensors adequate time to process the data read timely.

\section{ACKNOWLEDGMENT}

I would like to extend my profound gratitude to God Almighty for the opportunity to work under my supervisor in person of Assoc. Prof. Dr. Mohamed Hussein.

\section{REFERENCES}

[1] Y. Shan, W. Yang, C. Chen, J. Zhou, L. Zheng, and B. Li, "CF-Pursuit: A Pursuit Method with a Clothoid Fitting and a Fuzzy Controller for Autonomous Vehicles," International Journal of Advanced Robotic Systems, vol. 12, 2015.

[2] A. Ollero and O. Amidi, "Predictive path tracking of mobile robots. Application to the CMU Navlab," in Proceedings of 5th International Conference on Advanced Robotics, Robots in Unstructured Environments, ICAR, 1991, pp. 1081-1086.

[3] A. Garcia-Cerezo, A. Ollero, and J. Martinez, "Design of a robust high-performance fuzzy path tracker for autonomous vehicles," International journal of systems science, vol. 27, pp. 799-806, 1996.

[4] O. Amidi and C. E. Thorpe, "Integrated mobile robot control," in Fibers' 91, Boston, MA, 1991, pp. 504-523.

[5] N. Montés, M. C. Mora, and J. Tornero, "Trajectory generation based on rational bezier curves as clothoids," in Intelligent Vehicles Symposium, 2007 IEEE, 2007, pp. 505-510.

[6] K. O. Arras, N. Tomatis, B. T. Jensen, and R. Siegwart, "Multisensor on-the-fly localization:: Precision and reliability for applications," Robotics and Autonomous Systems, vol. 34, pp. 131-143, 2001.

[7] R. Wallace, A. Stentz, C. E. Thorpe, H. Maravec, W. Whittaker, and T. Kanade, "First Results in Robot RoadFollowing," in IJCAI, 1985, pp. 1089-1095.

[8] J. L. Martínez, A. Pozo-Ruz, S. Pedraza, and R. Fernandez, "Object following and obstacle avoidance using a laser scanner in the outdoor mobile robot Auriga$\alpha, "$ in Intelligent Robots and Systems, 1998. Proceedings., 1998 IEEE/RSJ International Conference on, 1998, pp. 204-209.

[9] R. C. Arkin, Behavior-based robotics: MIT press, 1998

[10] L. Scharf, W. Harthill, and P. Moose, "A comparison of expected flight times for intercept and pure pursuit missiles," IEEE Transactions on Aerospace and Electronic Systems, vol. 4, pp. 672-673, 1969.

[11] R. C. Coulter, "Implementation of the pure pursuit path tracking algorithm," DTIC Document1992.

[12] A. Ollero, A. García-Cerezo, and J. Martinez, "Fuzzy supervisory path tracking of mobile reports," Control Engineering Practice, vol. 2, pp. 313-319, 1994. 
[13] K. N. Murphy, "Analysis of robotic vehicle steering and controller delay," in Fifth International Symposium on Robotics and Manufacturing (ISRAM), 1994, pp. 631636.

[14] A. Ollero and G. Heredia, "Stability analysis of mobile robot path tracking," in Intelligent Robots and Systems 95.'Human Robot Interaction and Cooperative Robots', Proceedings. 1995 IEEE/RSJ International Conference on, 1995, pp. 461-466.

[15] A. Rodríguez-Castaño, G. Heredia, and A. Ollero, "Analysis of a GPS-based fuzzy supervised path tracking system for large unmanned vehicles," in Proceedings of the 4th IFAC International Symposium on Intelligent Components and Instruments for Control Applications (SICICA'00), 2000, pp. 141-146.

[16] J. L. Martínez, J. Morales, A. Mandow, and A. GarcíaCerezo, "Steering limitations for a vehicle pulling passive trailers," Control Systems Technology, IEEE Transactions on, vol. 16, pp. 809-818, 2008.

[17] T. Hellström, T. Johansson, and O. Ringdahl, "Development of an autonomous forest machine for path tracking," in Field and Service Robotics, 2006, pp. 603614.

[18] R. Gockley, J. Forlizzi, and R. Simmons, "Natural person-following behavior for social robots," in Proceedings of the ACM/IEEE international conference on Human-robot interaction, 2007, pp. 17-24.

[19] K. Petrinec, Z. Kovačić, and A. Marozin, "Simulator of multi-AGV robotic industrial environments," in Industrial Technology, 2003 IEEE International Conference on, 2003, pp. 979-983.

[20] C. Urmson, C. Ragusa, D. Ray, J. Anhalt, D. Bartz, T. Galatali, et al., "A robust approach to high-speed navigation for unrehearsed desert terrain," Journal of Field Robotics, vol. 23, pp. 467-508, 2006.

[21] A. Stentz, C. Dima, C. Wellington, H. Herman, and D. Stager, "A system for semi-autonomous tractor operations," Autonomous Robots, vol. 13, pp. 87-104, 2002.

[22] P. Zhao, J. Chen, Y. Song, X. Tao, T. Xu, and T. Mei, "Design of a control system for an autonomous vehicle based on Adaptive-PID," Int. J. Adv. Robot. Syst, vol. 9, 2012.

[23] S. Huang and G. Lin, "Parallel auto-parking of a model vehicle using a self-organizing fuzzy controller," Proceedings of the Institution of Mechanical Engineers, Part D: Journal of Automobile Engineering, vol. 224, pp. 997-1012, 2010.

[24] M. Abbas, "Non-linear model predictive control for autonomous vehicles," University of Ontario Institute of Technology, Ontario, Canada, 2011.

[25] J. Wit, C. D. Crane, and D. Armstrong, "Autonomous ground vehicle path tracking," Journal of Robotic Systems, vol. 21, pp. 439-449, 2004.

[26] J. Morales, J. L. Martínez, M. A. Martínez, and A. Mandow, "Pure-pursuit reactive path tracking for nonholonomic mobile robots with a 2D laser scanner," EURASIP Journal on Advances in Signal Processing, vol. 2009, p. 3, 2009.

[27] A. Pozo-Ruz, J. Martínez, and A. García-Cerezo, "Integration of a rotary sonar in the mobile robot RAM2," in Proceedings of the 3rd IFAC International Symposium on Intelligent Components and Instruments for Control Applications (SICICA'97), 1997, pp. 147151. 